\title{
Visualization of AMPA Receptor Distribution during Long Term Potentiation with Correlative Light and Electron Microscopy
}

\author{
Ye Sun ${ }^{1,3,4^{*}}$, Naomi Kamasawa ${ }^{2,4}$ and Ryohei Yasuda ${ }^{1,3,4}$ \\ ${ }^{1}$ Neuronal Signal Transduction Group, ${ }^{2}$ Electron Microscopy Core Facility, Max Planck Florida \\ Institute for Neuroscience, Jupiter, FL, USA. \\ ${ }^{3}$ International Max Planck Research School for Brain and Behavior, Jupiter, FL, USA. \\ ${ }^{4}$ FAU/Max Planck Florida Institute Joint Graduate Program in Integrative Biology and Neuroscience, \\ Florida Atlantic University, Boca Raton, FL, USA. \\ * Corresponding author: ye.sun@mpfi.org
}

Long-term potentiation (LTP) is a critical process in learning and memory. Dendritic spines, where neurons receive major excitatory inputs, are the fundamental structure and first line responder in LTP. A key to understand the mechanisms of LTP is to understand the synaptic molecules trafficking in this process. Light microscopy live imaging is a common technique used to monitor molecule trafficking [1]. However, due to diffraction limit, it is impossible to resolve molecule distribution within the tiny size of dendritic spines $\left(\sim 0.5 \mu \mathrm{m}^{3}\right)$ with regular light microscope. Electron microscopy (EM) holds the best resolution for structural visualization, but cannot capture functional features in the LTP process. Using correlative light and electron microscopy to bridge the gap, we manage to visualize distribution of AMPA receptors in dendritic spines in 3D EM captured immediately after spine specific LTP induction.

We cultured mouse hippocampal organotypic slices, and expressed SEP-GluR1 and SEP-GluR2 in a subpopulation of CA1 pyramidal neurons with gene gun transfection. 2-photon MNI-glutamate uncaging was applied to selected spines on a secondary dendrite to induce spine specific LTP (Figure 1A, B). Expansion of the spine head volume was recorded with 2-photon microscopy, and organotypic slices were then immediately fixed in $4 \%$ paraformaldehyde and $0.5 \%$ glutaraldehyde. To facilitate locating the same spines in EM, artificial marks were embedded by line-scan laser on top and bottom of target neurons, and along the stimulated secondary dendrites (Figure 1C). After cryo-protected with sucrose, slices were permeabilized by liquid nitrogen freeze and thaw cycles, and then immunolabed with anti-GFP antibody followed by nanogold conjugated secondary antibody. Silver enhancement, OsO4 post-fixation, uranyl acetate staining, and dehydration were performed before slices embedded in Durcupan resin. Serial sections were cut and collected onto Kapton tape with Automated Tape Collecting Ultramicrotome (ATUM), followed by scanning electron microscopy (SEM) imaging [2].

Under the guidance from laser burning marks and gold particles labeling pattern, targeted secondary dendrite can be identified after several rounds of imaging at increasing resolution. High resolution (4 $\mathrm{nm} /$ pixel) images were taken on serial sections to make 3D reconstruction of the stimulated spine and nearby control spines on the same dendrite to analyze the distribution of AMPA receptors (Figure 1D-F). As a result, we observed majority of the gold particles located along the plasma membrane. We focused our analysis in extrasynaptic area, since antibody labeling efficiency in synaptic region was greatly limited by the compacted synaptic structures. Structural LTP induced dramatic expansion of spine head volume $\left(\mathrm{LTP}=0.209 \pm 0.038 \mu \mathrm{m}^{3}\right.$, control $\left.=0.064 \pm 0.005 \mu \mathrm{m}^{3}\right)$, as well as the extrasynaptic membrane size measured in the $3 \mathrm{D}$ reconstructed models $\left(\mathrm{LTP}=2.939 \pm 0.413 \mu \mathrm{m}^{2}\right.$, control $\left.=1.178 \pm 0.082 \mu \mathrm{m}^{2}\right)$. Size of post-synaptic density (PSD) didn't show significant change (LTP $=2.375 \pm 0.563 \mu \mathrm{m}^{2}$, control $=1.753 \pm 0.192 \mu \mathrm{m}^{2}$ ). The amount of gold particles labeling for AMPA receptors in extrasynaptic area increased in proportion to the 
size of extrasynaptic membrane (AMPA receptor density in extrasynaptic area for $\mathrm{LTP}=1.070 \pm 0.078 / \mu \mathrm{m}^{2}$, control $=1.000 \pm 0.029 / \mu \mathrm{m}^{2}$ ). Some spines contained spine apparatus or postsynaptic vesicles. LTP spines had a lower percentage containing these structures compared to control spines ( 4 in 9 for LTP, 39 in 63 for control spines contained spine apparatus). Interestingly, the spine apparatus were all stained with AMPA receptors in LTP spines (Figure 1D), while rarely positive in control spines (5/39). In conclusion, this method allows visualization of AMPA receptor distribution in dendritic spine extrasynaptic region together with sub-spine structures, in a direct correlation with single spine activity history.

\section{References:}

[1] C Tardin et al., EMBO Journal, 22 (2003), p. 4656-4665.

[2] N Kamasawa et al,. Micros. Microanal. 21 (Suppl 3) (2015), p. 0635.
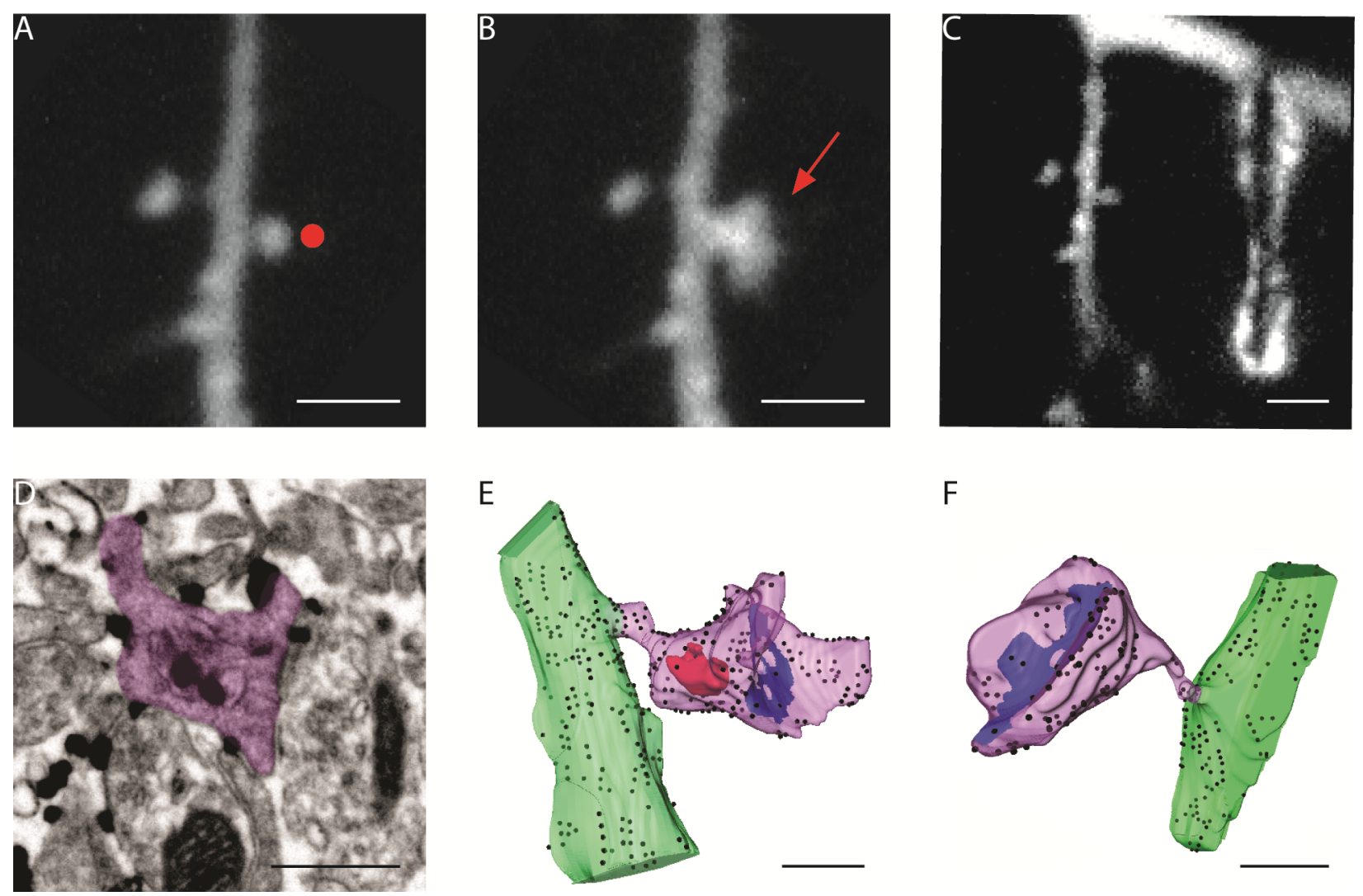

Figure 1. A segment of a secondary dendrite was selected for glutamate uncaging to induce spine specific structural LTP. Uncaging position is indicated in red dot in A, and expansion of spine head volume is shown in B (red arrow). A group of burning marks were made to label the position of target neuron and the target dendrite (C) to facilitate EM correlation. High resolution (4 nm/pixel) SEM images (D, spine colored in purple) were acquired to make reconstruction of the target spines (E) and control spines (F, green for dendrite, purple for spine head, blue for PSD, and red for spine apparatus). Gold particles are marked in black dots to visualize the AMPAR distribution in 3D. Scale bar: A-C, $2 \mu \mathrm{m}$; D-F, $0.5 \mu \mathrm{m}$. 